\title{
An Easy Management of Gingival Leukoplakia Using Cotton- Swab Nitrogen: A Case Report
}

\author{
Silvio Hirota, Norberto Sugaya and Dante Migliari* \\ Department of Stomatology, Oral Medicine Division, School of Dentistry, University of São Paulo, Brazil
}

\author{
Keywords \\ White plaque, Oral leukoplakia, Gingival manifestation, Liquid nitrogen
}

\section{Introduction}

Oral leukoplakia $(\mathrm{OL})$ is certainly the most relevant lesion that affects the oral mucosa, since it has an intrinsic potential for malignant transformation, specifically to squamous cell carcinoma (OSCC); and, besides, constitutes an important issue on clinical assessment. Thus far, no treatment (or management) can prevent the risk of malignant transformation of OL. It is said that surgical approach can reduce (but not eliminate) the odds of OL to develop into OSCC [1].

Biopsy is usually taken with the purpose to: a) To exclude other similar disease such as oral lichen planus or candida leukoplakia [2].; b) To disclose, if present, the extent of epithelial dysplasia; and c) To detect any superficial malignancy not yet clinically perceived $[1,3]$.

Small OL lesion can be removed by excisional biopsy and patient placed under periodical monitoring for either to detect recurrence, the development of a new lesion or, possibly, a malignant change $[1,3]$.

Therefore, no matter how is the efficacy of any surgical intervention, it does not preclude follow-ups. This article describes the use of cryosurgery for small OL lesions, in an area of low risk of malignant transformation.

\section{Case Report}

A 72-year-old white male was referred to our clinic for evaluation and management of a white lesion on his gingiva (Figure 1). The lesion was asymptomatic, of homogeneous pattern, measuring approximately $20 \mathrm{~mm}^{2}$, disclosed during a routine dental check-up. The patient was clinically healthy despite being a long-time smoker. He was not an alcoholic drinker; and was taking antihypertensive medication. The clinical diagnosis was of oral leukoplakia. Frictional keratosis was ruled out (there was no evidence of trauma-associated); candida leukoplakia [2] was excluded by a negative result for pseudohyphae on smear examination with $10 \%$ potassium hydroxide; an oral lichen planus lesion in plaque was possible but also ruled out since there was no white striae and, more importantly, the presence of other OLP lesions elsewhere in the oral mucosa, which are clinical requirements for a diagnosis of OLP in plaque.

As the lesion was small and with normal thickness for a keratotic plaque, a decision to use liquid nitrogen at -190 ${ }^{\circ} \mathrm{C}$ applied with a cotton swab was made. This technique consisted of initially placing the cotton swab into liquid nitrogen for a few seconds and then applying it over the lesion's surface until an ice ball is formed. Subsequently, as the cotton swab is removed, the ice is allowed to thaw normally. Usually three consecutive freeze-thaw cycles are required (Figure 2). Patient should be instructed to use a painkiller latter on the day if necessary. No recurrence has been seen after 10 months of follow-up. Patient is still being monitored on a 6-month interval basis.

\section{Discussion}

On clinical assessment, OL can be of two type, homogeneous (characterized by a smooth or wrinkled surface) and non-homogeneous (when its surface is mixed with read areas and/or exophytic proliferation).

The use of liquid nitrogen (more often called cryosurgery) in some flat oral lesions is very convenient. First, no anesthesia injection is required; the application is easy and almost no medication (painkiller) deems necessary during the healing process. Under a very low temperature, the liquid nitrogen acts by locally freezing and destroying the tissue surface in

*Corresponding author: Dante Migliari, DDS, PhD, Departamento de Estomatologia, Faculdade de Odontologia, Universidade de São Paulo, Av. Prof. Lineu Prestes, 2227, Cidade Universitária, São Paulo, SP, 05508-000, Brazil, Tel: +55-11-94383-7972

Accepted: October 12, 2019

Published online: October 14, 2019

Citation: Hirota S, Sugaya N, Migliari D (2019) An Easy Management of Gingival Leukoplakia Using Cotton-Swab Nitrogen: A Case Report. Dermatol Arch 3(1):86-88 


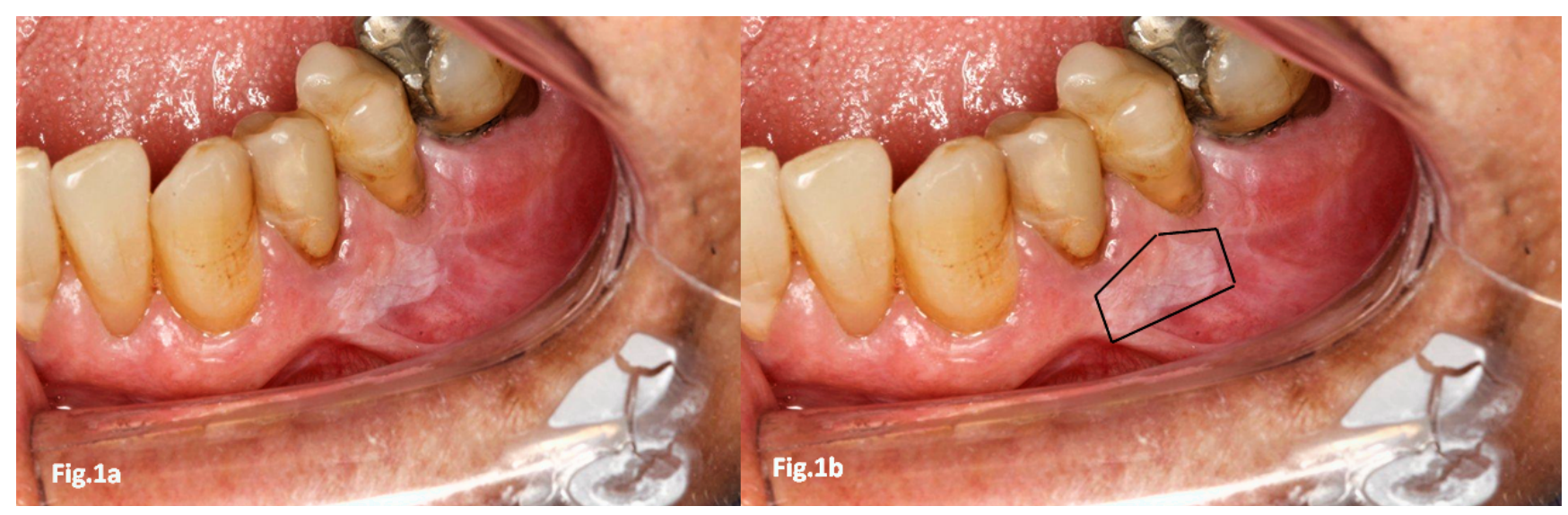

Figure 1: A homogeneous white plaque on the gingival mucosa, a characteristic clinical presentation of oral leukoplakia.

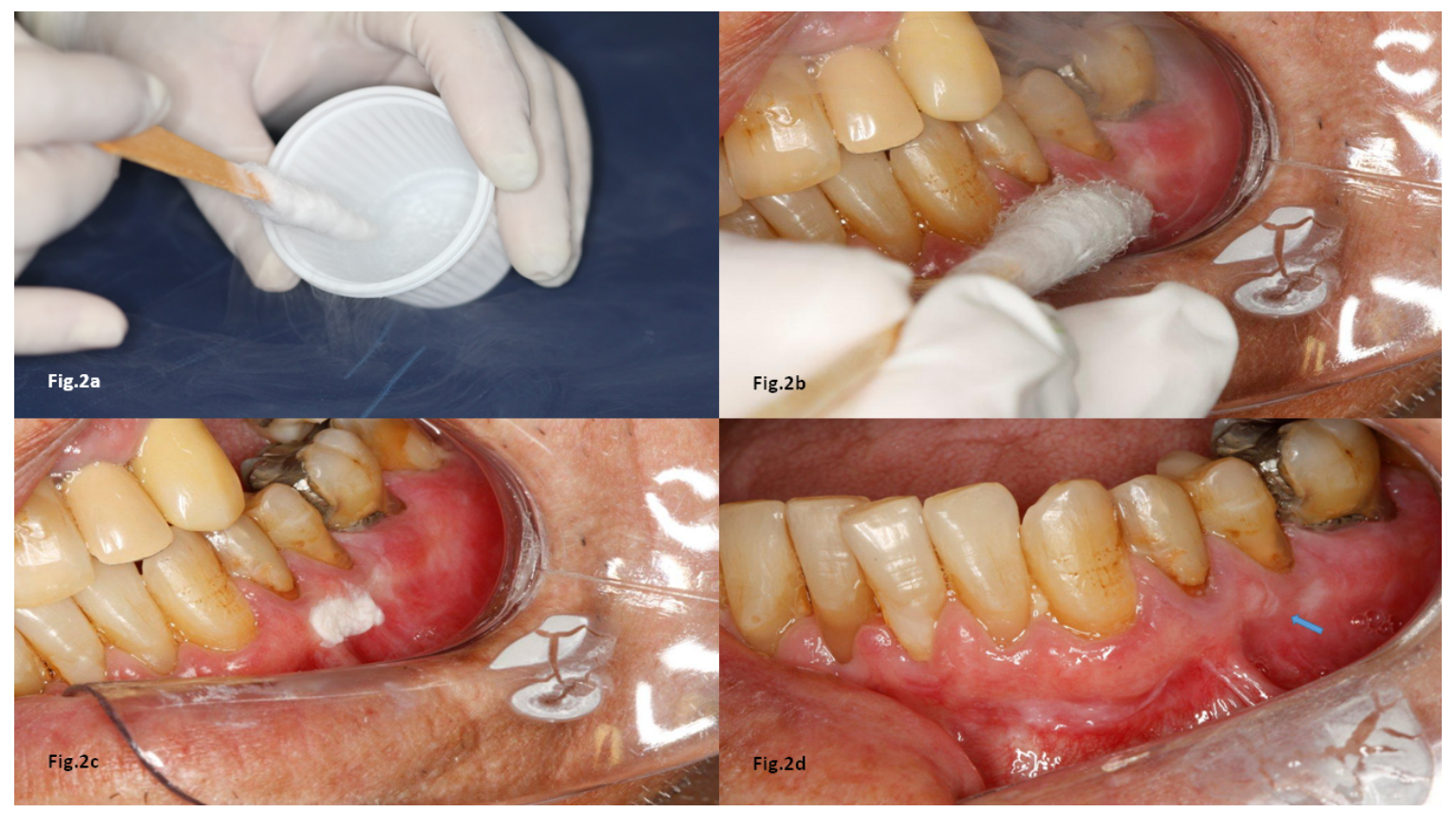

Figures 2: a) Immersing a cotton swab into liquid nitrogen; b) Applying the frozen swab cotton over the lesion; c) Letting the frozen lesion's surface thaw normally; d) A photograph taken one month later showing a slight scar (arrow).

situ [4]. Opposing to its use, it has been argued this technique lacks histopathological examination. This would be true if the lesion was of non-homogeneous type (showing redness, proliferation or with a significant thickness, and, additionally, located in high-risk areas, such as tongue's lateral and ventral surfaces, and floor of the mouth, or soft palate).

It has also been argued that this technique (cotton swab) can be used in extensive, non-homogeneous OL lesion following a biopsy procedure with its respective histopathological examination $[5,6]$. In this respect, the use of high-power laser has taken precedence over cryosurgery, with better results in term of lower recurrence rate and, moreover, by reaching deeper areas of the lesion as well as over of its clinical margin [7].

The present case did not have any signs of aggressiveness. Hence, a noninvasive procedure was chosen, with a successful outcome in terms of effectively removing the le- sion and, so far, without recurrence. The monitoring for OL is always mandatory, regardless the technique employed.

The very low temperature reached by liquid nitrogen produces lysis of the keratin along with the superficial subjacent tissue. The healing process using this technique occurs usually in a very satisfactory way. There was some scar (arrow on Figure $2 \mathrm{~d}$ ); nevertheless without a functional or aesthetic interference.

\section{References}

1. Dost F, Lê Cao K, Ford PJ, et al. (2014) Malignant transformation of oral epithelial dysplasia: A real-world evaluation of histopathologic grading. Oral Surg Oral Med Oral Pathol Oral Radiol 117: 343-352.

2. El-Naggar AK, Chan JKC, Grandis JR, et al. (2017) WHO classification of head and neck tumours. ( $4^{\text {th }}$ edn), IARC Publications, France. 
3. van der Waal I (2009) Potentially malignant disorders of the oral and oropharyngeal mucosa; Terminology, classification and present concepts of management. Oral Oncol 45: 317-323.

4. Farah CS, Savage NW (2006) Cryotherapy for treatment of oral lesions. Aust Dent J 51: 2-5.

5. Yu CH, Chen HM, Chang CC, et al. (2009) Cotton-swab cryothera- py for oral leukoplakia. Head Neck 31: 983-988.

6. Gongloff RK, Samit AM, Greene GW Jr, et al. (1980) Cryosurgical management of benign and dysplastic intraoral lesions. J Oral Surg 38: 671-676.

7. Van der Hem PS, Nauta JM, van der Waal JE, et al. (2005) The results of $\mathrm{CO} 2$ laser surgery in patients with oral leukoplakia: A 25 year follow up. Oral Oncol 41: 31-37. 